\title{
Mediatory cue proximity and mental subnormality in the perception of rectilinear dot progressions ${ }^{1}$
}

\author{
EDWARD A. HOLDEN, JR. ${ }^{2}$ \\ JOHNSTONE TRAINING AND RESEARCH CENTER
}

\begin{abstract}
This study investigated the relationship between illusory displacement of sequentially presented light stimuli and their proximity to mediatory cues. Twenty-four retardates, 24 equal $C A$ normals, and 24 equal MA normals were tested to determine the relative stabilizing effects of four different frame conditions on these groups. Illusory displacement decreased from least to most proximate cue condition, was least for the $M A$ group, and was most for retardates. It was conchuded that interstimulus mediation referents, rather than restricted eye movement, facilitated veridical perception of successive light positions.
\end{abstract}

Under subdued illumination and homogeneous visual field conditions, if spatially distributed point sources of light are illuminated in a straight line, Ss frequently experience an illusory displacement of the lights in an erratic manner about their actual progression lines (Holden, 1966). Unlike autokinetic movement, however, there is no apparent drifting of individual lights while they are illuminated. Rather, the lights are displaced in relation to each other. When, for example, the third light in a horizontal array is illuminated it might appear above or below an imaginary line drawn through the first two lights. Further investigations have demonstrated that increasing the time between lights or decreasing locus redundancy ${ }^{3}$ of the progression lines increases illusory displacement (Holden, $1967 \mathrm{a})$, but that stimulus duration (Holden, 1968), stimulus intensity (Holden, 1969) and eye movement (Holden, 1967b) are not relevant parameters. This series of studies has been reviewed by Holden (1970).

racilitation of illusory displacement with increasing interstimulus interval (ISI) was interpreted to reflect progressively greater dissipation of the locus-defining trace from successive lights, continuity between which is necessary for veridical perception of their objective relationships. For the greater displacement obtaining from decreased locus redundancy, first consideration was given to the possible role of eye movement, since there is experimental evidence (Leyzorek, 1951; Matin \& Kibler, 1966; Matin, Pearce,
Matin, \& Kibler, 1966) that in complete darkness the perceived uniplanar spatial relationship between two sequential light stimuli, $S_{1}$ and $S_{2}$, is affected in a predictable manner by the direction and extent of intervening eye movement. If, for example, $S$ 's eyes were to drift upward between $S_{1}$ and $S_{2}$ in one of the horizontal light sequences, $S_{2}$ would appear displaced below its objective relation to $S_{1}$.

Although the Johnstone laboratory studies were not conducted in complete darkness, the low ambient light level and relatively homogeneous visual field conditions suggested that part of the illusory displacement phenomenon may have derived from interstimulus eye movements. It was assumed that S's perception of the relative positions of temporally distributed visual stimuli depends upon information from two sources (1) locus trace, defined by the display screen, $X, Y$, coordinates (these coordinate values derive from textural and other invariant stimuli associated with the display screen and function as spatial anchors during the interstimulus interval); and (2) retinal signal, defined by the retinal position of any stimulus in the sequence relative to that of an immediately preceding stimulus. Retinal signal is assumed to be determined by $S_{1}$ and $S_{2}$ retinal positions at the onset of $S_{2}$.

On the assumption that the high redundancy (HR) series of light sequences effected closer proximity between eye fixations and their antecedent stimulus loci (according to the principles of contiguity learning, more frequent repetition of the same light sequence effected stronger association between each light position and its defining $X, Y$, values), greater illusory displacement under the low redundancy (LR) condition was hypothesized to have resulted from the larger eye movements occasioned by successive stimuli. Subsequent experimental tests of this hypothesis demonstrated no differential effect among three levels of eye movement, however (Holden, 1967b). The degree of illusory displacement did not differ among Ss who judged rectilinear dot progressions under the following conditions: (1) fixation point, adjacent lights 1 in. apart; (2) no fixation point, adjacent lights $1 \mathrm{in}$. apart; and (3) no fixation point, adjacent lights 2 in. apart. Hence, it was altematively concluded that in the $H R$ condition textural and other invariant stimuli associated with the pattern display screen were, by repeated and predictable association with the same light sequence on each trial, more effectively utilized as interstimulus referents for successive light positions. Independent of its effect upon eye movement, repetition of the same light sequence on each trial provided stronger associations between the light stimuli and textural cues that served as spatial anchors during the interstimulus intervals.

The primary purpose of the present study was to test the hypothesis that if interstimulus representations of light positions depend upon the associative strength between each light and its defining $X, Y$, parameters, then increasing association potential of the display-screen cues should increase their mediative efficacy. Accordingly, it was predicted that mediatory potential would vary with cue proximity, which, in turn, would vary with the size of frames surrounding the central matrix. Consequently, the number of "straight" judgments should increase progressively from a no-frame (NF) condition through a large-frame (LF), medium-frame (MF), and small-frame (SF) condition.

The second purpose of this study was to compare mental retardates, equal CA normals, and equal MA normals with respect to their abilities to utilize the different degrees of mediatory reference cues. On the basis of three previous studies (Holden, 1967a, 1969; Belmont \& Butterfield, 1969), which, with one minor exception, demonstrated no differences between trace continuity for retardates and normals in the illusory displacement task, it was predicted that the three groups would not differ in the number of "straight" responses for dot progressions without a surrounding frame. No predictions were made about the relative performances of the three groups under the three increased cue-proximity conditions.

Mubjects
The Ss were 24 educable retardates
$(\overline{C A}=15.97, \quad S D=1.42, \quad \overline{I Q}=64.46$,
SD $=9.12)$ in residence at the Edward $R$.



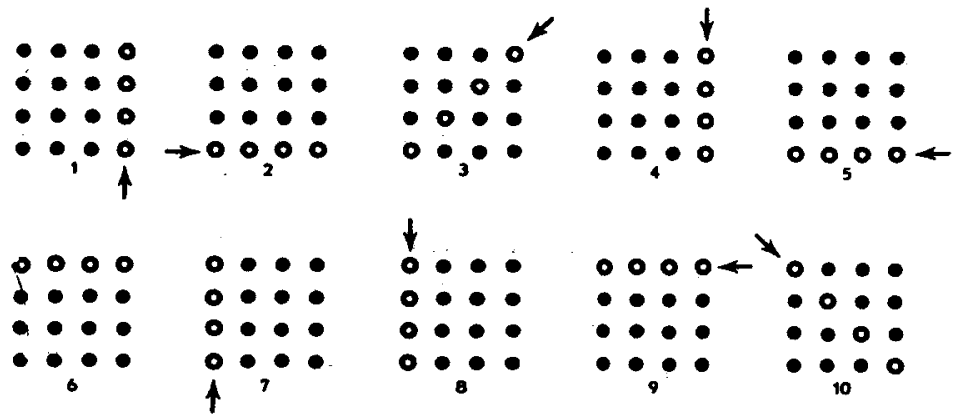

Johnstone Training and Research Center, 24 equal iA normals $(\overline{C A}=16.34$, $\mathrm{SD}=.79$ ) from a neighboring high school, and 24 equal $M A$ normals $(\overline{C A}=9.49$, $\mathrm{SD}=.20$ ) from a neighboring elementary school. Males and females were equally represented in each group. IQ data were not available for the $C A$ and MA groups, but average IQs for 108 Ss tested from the same schools in previous years were $\mathbf{1 0 4 . 9 5}$ and 103.61 , respectively.

\section{Apparatus and Stimuli}

Essentials of the apparatus are described in an earlier report (Holden, 1965). The task required $\mathrm{Ss}$ to judge the rectilinearity of straight dot progressions generated by sequentially illuminating four lights within a $4 \times 4$ matrix of neon lamps (NE-2s). The 10 dot progressions, each appearing as it would were all lamps illuminated simultaneously, are shown by the open circles in Fig. 1. Arrows indicate the first light and direction of subsequent lamp illuminations for each sequence. Association potential of the display system was varied by presenting the 10 light sequences in Fig. 1 under four different frame conditions, viz, no frame, large frame (36 lamps), medium frame (28 lamps), and small frame (20 lamps) surrounding the central matrix. The four sides of these frames were, respectively, 3 , 2 , and 1 in. from the matrix, and contiguous lamps were spaced 1 in. between centers. The NF condition was identical to display-screen conditions in the preceding studies and may be assumed to have provided least opportunity for interstimulus anchoring of the sequential light positions. The entire display screen was covered with translucent black muslin so that the unilluminated lamps could not be seen. Frames were illuminated $3 \mathrm{sec}$ before onset of the first light in each sequence and remained on until $3 \mathrm{sec}$ after termination of the last light. Sequence lights were illuminated for $0.1 \mathrm{sec}$ and were separated by $3.0-\mathrm{sec}$ intervals.

\section{Procedure}

The Ss were tested individually in sessions requiring approximately $20 \mathrm{~min}$, during which time they viewed the dot progressions binocularly from a position $6 \mathrm{ft}$ directly in front of the lamp matrix. At this distance, adjacent lamps in the vertical and horizontal progressions subtended a visual angle of $48 \mathrm{~min}$, and for adjacent lamps in the diagonal progressions the angle was $1 \mathrm{deg} 8 \mathrm{~min}$. A pretest, details of which have been described elsewhere (Holden, 1968), was given at the beginning of each session to insure that Ss could discriminate between rectilinear and nonrectilinear sequences when the line stimuli were presented simultaneously.

After the pretest, Ss were told that they would see four dots of light flash on and off, one after the other, on the pattern-display screen, and that after each sequence they should tell $\mathrm{E}$ whether the lights flashed in a straight line or in a crooked line. They were also told that on some trials a frame of lights would precede the dot sequence, and each of the three frames was demonstrated for a few seconds.

The Ss received 40 trials, consisting of all possible combinations of the 10 dot progressions and four frame conditions. These combinations were randomized within two blocks of 20 trials so that each progression and frame condition occurred an equal number of times in both blocks. Six males and six females from each $S$ group received Block 1 followed by Block 2, the remaining Ss receiving these blocks in reverse order.

\section{RESULTS AND DISCUSSION}

The mean number of "straight" judgments associated with each frame condition for the three groups are plotted in Fig. 2. A Type I variance analysis (Lindquist, 1953) of these curves demonstrated a significant group effect $(F=4.60, \quad \mathrm{df}=2 / 69, \quad \mathrm{p}<.025), \quad \mathrm{a}$ significant frame effect $(F=62.01$, $\mathrm{df}=3 / 207, \mathrm{p}<.001$ ), and a nonsignificant interaction.

Multiple range tests (Duncan, 1955) of frame effect within each subject category showed the SF consistently to have produced significantly more "straight" judgments than any of the other frame
Fig. 1. Progressions of lamp illuminations on the 10 dot sequences.

conditions ( $p<.01)$, but, except for the NF vs LF condition in the equal MA group $(p<.01)$, no other differences were significant. Multiple range tests between $S$ categories revealed the following results: (1) For combined frame conditions, a significant difference $(p<.01)$ between the retardate and equal MA groups; (2) within the NF condition, no significant difference between any of the groups; (3) within the LF condition, a significant difference between the retardate and $(p<.01)$ equal MA groups; $(4)$ within the MF condition, a significant difference between the equal MA group and both the equal $C A$ and retardate groups $(p<.05)$; and (5) within the SF condition, a significant difference between the retardate and equal $C A$ normal groups $(\mathrm{p}<.05)$.

Results for the SF condition lend support to the hypothesized greater mediative efficacy obtaining from increased association potential of the display-screen cues. As one possible explanation for the weak effects of the LF and MF conditions it can be conjectured that their proximity was too remote to provide additional associative strength over that available from the NF condition, in which the screen border provided the most proximate reference cues.

The present findings, in conjunction with those of Leyzorek (1951), Matin and Kibler (1966), Matin, Pearce, Matin, and Kibler (1966), and Holden (1967b), permit the conclusion that eye movement is an important determinant of visual space direction only when no visual frame of reference is available. It appears that when

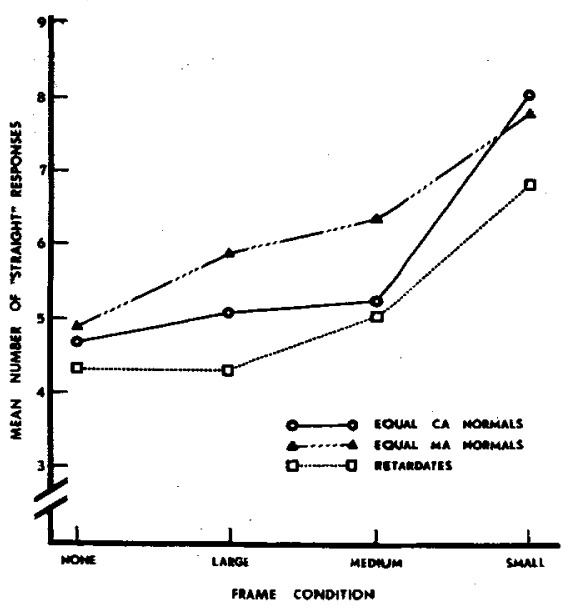

Fig. 2. Mean number of "straight" responses per frame condition for each group. 
reference cues are available, any ocular deviation from $S_{1}$ during the ISI is encoded so that the relative position of $S_{2}$ remains veridical.

Failure to find differences between $S$ groups under the NF condition is as predicted and further substantiates findings from previous studies using dot progressions and homogeneous visual-field conditions for comparing stimulus trace processes in retardates and normals (Holden, 1970). The only consistent group effect was the inferior performance of the retardates when compared with the equal MA normals under the LF and MF conditions and with the equal CA normals under the SF condition, indicating that they were less able to utilize the supplemental mediatory cues as efficiently as could the normals. This less efficient utilization may relate to Ellis's 1969 theory of rehearsal strategy deficits in mental defectives.

Explanation of the intermediate position of the equal CA group under the LF and MF conditions is highly conjectural, since they do not differ from the equal MA normals under the NF and SF conditions. Possibly the older normal group used a different strategy from the younger normal group under the two least proximate frame conditions. For example, Hayes and King (1967) and also Winters (1969) have noted that in older normals observer biases may influence responses.

Since the NF condition in the present study was identical to that of the $3.0-\mathrm{sec}$ LR condition of the locus-redundancy study (Holden, 1967a), any disparity between the effect of increased locus redundancy vs cue supplementation on the illusory displacement phenomenon should appear as a difference between performance under the 3.0-sec $H R$ and SF conditions, respectively. As a baseline reference, the 3.0-sec LR condition and NF condition were compared for each $\mathrm{S}$ group. The mean number of "straight" responses was significantly higher for retardates in the present study $(t=2.59, \mathrm{df}=38$, $p<.02)$, but this difference can be accounted for by the spuriously depressed performance of the retardates under the 3.0-sec ISI in the earlier study (cf. Belmont \& Butterfield, 1969). For each S group, a comparison was then made between the 3.0-sec HR condition of the earlier study and the SF condition of the present one. In all groups, the SF condition gave significantly more "straight" judgments $\left(t_{C A}=2.92, d f=38, p<.01 ; t_{M A}=3.73\right.$, df $=38, \quad p<.001 ; \quad t_{R}=2.25, \quad$ df $=38$, $p<.05$ ), thereby demonstrating greater efficacy of the enriched visual frame of reference over increased locus redundancy. These comparisons should be interpreted with reservations, however, since it has been demonstrated (Holden, 1968) that the effect of an experimental treatment in this task is greater when different levels of the variable operate within the same experimental session than when they operate at widely disparate times.

\section{REFERENCES}

BELMONT, J. M., \& BUTTERFIELD, E. C. The relations of short-term memory to development and intelligence. In L. P. Lipsitt and $\mathrm{H}$. Reese (Eds.), Advances in child development and behavior. Vol. 4. New York: Academic Press, in press.

DUNCAN, D. B. Multiple range and multiple F tests. Biometrics, 1955, 11, 1-42.

ELLIS, N. R. Memory processes in retardates and normals: Theoretical and empirical considerations. In N. R. Ellis (Ed.), International review of reseurch in mental retardation. Vol. 4. New York: Academic Press, 1969, in press.

HAYES, C. J. A., \& KING, W. L. Two types of phenomenal instructions for size and distance judgments of objects presented in a two-dimensional plane. Perception \& Psychophysics, 1967, 2, 556-558.

HOLDEN, JR., E. A. Temporal factors and subnormality in visual pattern recognition: $A$ test of stimulus trace theory. Journal of Comparative \& Physiological Psychology, $1965,59,340-344$.

HOLDEN, JR, E. A. Stimulus duration and subnormality in visual pattern recognition: $A$ further test of stimulus trace theory. Joumal of Comparative Physiological Psychology, $1966,62,167-170$.

HOLDEN, JR., E. A. In terstimulus in terval, locus redundancy, and mental subnormality in perception of rectilinear dot progressions. Journal of Comparative \& Physiological Psychology, 1967a, 64, 366-370.

HOLDEN, JR., E. A. Eye movements and the perception of rectilinear dot progressions. Psychonomic Science, 1967b, 7, 219-220.

HOLDEN, JR., E. A. Stimuhus duration and the perception of rectilinear dot progressions in educable retardates. American Journal of Mental Deficiency, 1968, 72, 599-602.

HOLDEN, JR., E. A. Stimulus intensity and mental subnormality in perception of rectilinear dot progressions. American Journal of Mental Deficiency, 1969a, 73, 749-752.

HOLDEN, JR., E. A. Sequential dot presentation measures of stimulus trace in retardates and nomals. In N. R. Ellis (Ed.), International review of research in mental retardation. Vol. 5. New York: Academic Press, 1970.

LEYZOREK, M. Two-point discrimination in visusl space as a function of the temporal interval between stimuli. Journal of Experimental Psychology, 1951, 41, 364-375.

LINDQUIST, E. F. Design and analysts of experiments in psychology and education. Boston: Houghton Mifflin, 1953.

MATIN, L., \& KIBLER, G. Acuity of visual perception of direction in the dark for various positions of the eye in orbit. Perceptual \& Motor Skills, 1966, 22, 407-420.

MATIN, L., PEARCE, D., MATIN, E., \& KIBLER, G. Visual perception of direction in the dark: Roles of local sign, eye movements, and ocular proprioception. Vision Research, $1966,6,453-469$.

WINTERS, J. J. A comparison of normals and retardates on physiological illusions. American Journal of Mental Deficiency, 1969, 73 956-962.

\section{NOTES}

1. The author extends sincere appreciation to Dr. Ralph Osborne, superintendent of the Hamilton Township school system, Mr. George Murphy, principal of Hamilton High School West. and Mrs. Isabel Robinson, principal of Kuser School, for their generous cooperation in providing students and testing space for this experiment.

2. Address: Edward R. Johnstone Training \& Research Center, Bordentown, New Jersey 08505.

3. In the low redundancy (LR) condition, sequences started at several different points and progressed in different directions, as opposed to : high-redundancy (HR) condition in which th same sequence of lights was presented on ear trial.

(Accepted for publication October 15, 1969.) 